\title{
Enhancement of aerobic fitness improves social functioning in individuals with schizophrenia
}

\author{
D. Kimhy ${ }^{1,2}$ D $\cdot$ C. Tay ${ }^{1} \cdot$ J. Vakhrusheva ${ }^{3} \cdot$ K. Beck-Felts $^{1} \cdot$ L. H. Ospina ${ }^{1} \cdot$ C. Ifrah ${ }^{1} \cdot$ M. Parvaz ${ }^{1} \cdot$ J. J. Gross $^{4}$. \\ M. N. Bartels ${ }^{5}$
}

Received: 26 August 2020 / Accepted: 2 December 2020 / Published online: 3 January 2021

c) Springer-Verlag GmbH Germany, part of Springer Nature 2021

\begin{abstract}
Individuals with schizophrenia display substantial deficits in social functioning (SF), characterized by chronic, lifelong presentations. Yet, at present there are few effective interventions to enhance SF in this population. Emerging evidence from studies of clinical populations that display similar SF deficits suggests that aerobic exercise (AE) may improve social skills. However, this putative impact has not been investigated in schizophrenia. Employing a single-blind, randomized clinical trial design, 33 individuals with schizophrenia were randomized to receive 12 weeks of Treatment-As-Usual (TAU; $n=17$ ) or TAU + AE $(n=16)$ utilizing active-play video games (Xbox 360 Kinect $)$ and traditional AE equipment. Participants completed an evaluation of aerobic fitness $\left(\mathrm{VO}_{2} \mathrm{max}\right)$ as well as self-, informant-, and clinician-reported SF measures at baseline and after 12 weeks. Twenty-six participants completed the study (79\%; TAU =13; AE =13). At follow-up, the AE participants improved their $\mathrm{VO}_{2}$ max by $18.0 \%$ versus $-0.5 \%$ in the controls (group $\mathrm{x}$ time interaction, $\mathrm{F}_{1,24}=12.88 ; p=.002$ ). Hierarchical stepwise regression analyses indicated improvements in $\mathrm{VO}_{2} \mathrm{max}$ significantly predicted enhancement in $\mathrm{SF}$ as indexed by self-, informant-, and clinician-reported measures, predicting $47 \%, 33 \%$, and $25 \%$ of the variance, respectively (controlling for baseline demographics, medications, mood symptoms, and social networks). Compared to the TAU group, AE participants reported significant improvement in SF (23.0\% vs. $-4.2 \%$; group $\times$ time interaction, $\left.F_{1,24}=7.48, p=.012\right)$. The results indicate that $\mathrm{VO}_{2}$ max enhancement leads to improvements in $\mathrm{SF}$ in people with schizophrenia. Furthermore, low $\mathrm{VO}_{2}$ max represents a modifiable risk factor of $\mathrm{SF}$ in people with schizophrenia, for which $\mathrm{AE}$ training offers a safe, non-stigmatizing, and nearly side-effect-free intervention.
\end{abstract}

Keywords Schizophrenia $\cdot$ Psychosis $\cdot$ Social fucntioning $\cdot$ Aerobic exercise $\cdot$ Aerobic fitness $\cdot$ Randomized clinical trial

D. Kimhy

david.kimhy@mssm.edu

1 Department of Psychiatry, Icahn School of Medicine at Mount Sinai, One Gustave L. Levy Place, Box 1230, New York, NY 10029, USA

2 MIRECC, The James J. Peters VA Medical Center, Bronx, NY, USA

3 Department of Psychiatry, Columbia University, New York, NY, USA

4 Department of Psychology, Stanford University, Stanford, CA, USA

5 Department of Rehabilitation Medicine, Albert Einstein College of Medicine, Bronx, NY, USA

\section{Background}

Individuals with schizophrenia display substantial deficits in social functioning (SF) [1]. These deficits, which often predate the onset of psychosis $[2,3]$, are characterized by chronic, lifelong presentations [4-7] that have been linked to heightened experiences of stigma [8], diminished quality of life, and disability [9]. Thus, poor SF represents a public health concern $[10,11]$ and an important target for interventions in this population [12].

Consistent with this view, interest in the development of interventions aiming to ameliorate poor SF in schizophrenia and related disorders has grown substantially in recent years [13]. A number of therapeutic approaches have been employed including cognitive remediation, cognitive behavioral therapy (CBT), third-wave therapies, as well as pharmacotherapy. However, evidence supporting the efficacy 
of these interventions has been mixed. A meta-analysis of cognitive remediation interventions for individuals with early psychosis indicated a small but significant effect size [14]. Yet, a recent meta-analysis of studies in individuals at clinical high-risk for psychosis reported no significant improvement in at 2- to 3-month follow-ups [15]. Interventions for individuals with schizophrenia employing CBT models have shown more promise. A meta-analysis indicated a large positive effect of CBT on SF [16], though many of the included studies were of low quality [16]. A more recent meta-analysis evaluating the effectiveness of CBT for psychosis showed small, but significant effect sizes for functioning by end-of-trials that became non-significant at follow-ups [17]. Likewise, recent findings suggest thirdwave cognitive-behavioral interventions for individuals with schizophrenia may be helpful, albeit with small effect sizes, as has been demonstrated in a recent meta-analysis [18]. Finally, pharmacological interventions have demonstrated limited efficacy in ameliorating poor SF in schizophrenia. Results from the CATIE trial, a large randomized clinical trial that followed-up participants for up to 18 months, noted modest improvements in SF, with no differences in ratings after 6,12 , or 18 months [19]. Altogether, these findings indicate a limited to modest efficacy of available treatments for poor SF in schizophrenia. Hence, while efforts to address social dysfunction in this population have increased over the past decade, progress to date has been modest, and there remains a critical need to identify and develop new treatment approaches to target poor SF in schizophrenia.

One approach that has been shown to enhance SF among other clinical populations presenting with social deficits is aerobic exercise training (AE). A meta-analysis of $16 \mathrm{AE}$ studies of individuals with autism spectrum disorder indicated significant improvements in social skills [20], with jogging and swimming being the most frequently applied therapeutic exercise activities. Specifically, social interaction skills showed significant benefits, with an overall average improvement of $39.1 \%$ [20]. Similarly, a systematic review of research on exercise interventions in individuals with autism spectrum disorder found significant improvements in social-emotional functioning outcomes [21].

Germane to schizophrenia, interest in employing physical exercise training to target clinical symptoms in this population has expanded substantially over the past two decades, as evident by the number of publications in PubMed (2000:12 $\rightarrow 2019: 110$ ). Individuals with schizophrenia, as well as those at clinical high risk of psychosis, tend to display highly sedentary lifestyles, resulting in poor aerobic fitness [22-24]. The bulk of the recent literature has centered on the impact of AE on neurocognition-findings from randomized clinical trials (RCT) by our group and others have demonstrated that AE leads to significant neurocognitive benefits [25-28], with improvements linked to exercise training fidelity [29].
Recent findings suggest AE may potentially benefit SF as well. Specifically, AE has been found to significantly improve a number of correlates of SF-a meta-analysis by Firth and colleagues [25] reported AE significantly improved social cognition, a predictor of real-world functioning [30]. Likewise, Dauwan et al. [31] found exercise training was superior to control conditions in improving negative symptoms, many of which index elements of social and affective functioning. Benefits were also noted for quality of life and global functioning. Consistent with these findings, Kern and colleagues [32] found improvements in social functioning were significantly related to gains in cardiorespiratory fitness $(r=.42 ; p<.01)$ among 54 Veterans aged $40-65$ years old with schizophrenia who were randomized to participate in a 12 week of $\mathrm{AE}$ or a non-aerobic stretching exercise intervention. Trend-level improvements in SF were seen in the $\mathrm{AE}$ group $(p<.09)$, as well as trend level difference in the between-group comparison ( $p<.06$; Cohen's $d=.35$ ). However, at present, there are no studies directly examining the potential impact of AE on SF in schizophrenia. Furthermore, findings from previous reports are limited by reliance on clinician-administered measures of SF, which may potentially have poor ecological validity, with virtually no direct input from patients or informants/caregivers.

To address this gap in the literature, our aim was to examine the impact of AE on SF in individuals with schizophrenia, employing comprehensive, multi-method evaluations of SF indexed by self-, informant-, and clinician-reports. We hypothesized that AE-related increases in aerobic fitness would predict improvement in SF among individuals with schizophrenia.

\section{Methods}

\section{Participants}

Data were obtained from a pilot RCT conducted at a large hospital (ClinicalTrials.gov Identifier NCT01897064). The medical center's institutional review board approved the study, and all participants provided written informed consent. Participants were recruited from outpatient mental health clinics in the greater New York City area. The inclusion criteria were a DSM-IV diagnosis of schizophrenia or related disorders; age 18-55 years; English-speaking; taking antipsychotic medication for at least 8 weeks and on current doses for 4 weeks and/or injectable depot antipsychotics with no change in the last 3 months; and medically cleared by a physician to take part in AE training. The exclusion criteria were a DSM-IV diagnosis of alcohol/substance abuse within the past month or alcohol/substance dependence within the past 6 months; recent use of street drugs (confirmed by a urine toxicology test); a history of seizures/ 
head trauma with loss of consciousness resulting in cognitive sequelae/rehabilitation; significant clinical abnormalities in physical examination, electrocardiogram, or lab assessments; untreated hyper- or hypothyroidism; extreme obesity (BMI $\geq 40$ ); being pregnant/nursing; having serious suicidal/homicidal risk; presence of moderate or more severe disorganization (SAPS global positive formal thought disorder $\geq 3$ ); more than a mild level of depressive symptoms (BDI $>18)$; and participation in a study involving neurocognitive assessment in the previous 3 months. All data were collected between May 2012 and July 2014.

Sample Characteristics: Forty-one individuals consented to participate in the study. Eight participants discontinued their participation prior to randomization due to difficulties keeping research appointments $(n=3)$, psychotic exacerbation $(n=1)$, diagnosis of cancer $(n=1)$, discovery of a benign brain tumor $(n=1)$, BDI $>18(n=1)$, and conflict with changed school schedule $(n=1)$. The remaining 33 individuals were randomized $(\mathrm{AE}=16, \mathrm{TAU}=17)$ of which 26 completed the study (79\%). In the AE group, all three non-completers dropped-out during the first week of the $\mathrm{AE}$ training after 0,1 , and 3 sessions, respectively, due to loss of contact $(n=1)$, long commute-time to $\mathrm{AE}$ site $(n=1)$, and not liking the AE program $(n=1)$. In the TAU group, four participants dropped-out due to relocation $(n=1)$, hypomanic episode $(n=1)$, and protocol violations $(n=2)$. The status of 3 of the 33 randomized participants (9\%) became un-blinded to raters - 1 in the TAU group (self-disclosure) and 2 in the $\mathrm{AE}$ group (1 self-disclosure, 1 accidental disclosure by clinical staff). Of note, we did not exclude participants who were prescribed beta-blockers or other medications known to influence heart rate out of concern that such exclusion would result in a non-representative sample as individuals with schizophrenia may be prescribed such medications for cardiac and/or other problems (e.g., akathisia). Only 2 out of the 33 randomized participants (6\%) were prescribed beta-blockers.

\section{Measures}

\section{Clinical assessments}

Diagnoses were established using The Structured Clinical Interview for DSM-IV. Clinical symptoms were assessed using the Scales for Assessment of Positive and Negative Symptoms (SAPS/SANS) [33, 34] and the Beck depression and anxiety inventories (BDI; Beck Depression Inventory, BAI; Beck Anxiety Inventory) [35, 36].

\section{Aerobic fitness}

Aerobic fitness was determined by a cardiopulmonary exercise test to establish $\mathrm{VO}_{2}$ peak, an index of the maximum capacity of an individual's body to transport and use oxygen during incremental AE activity. All tests were completed on weekdays at $\sim 10$ am and were performed on an electronically braked cycle ergometer [37] with a Viasys Encore metabolic cart [38]. The equipment was calibrated prior to every test. Continuous 12-lead telemetry was monitored via CardioSoft electrocardiogram software [39]. Participants completed a 5-min resting baseline, a 3 min of no-resistance warm-up, and a ramping exercise protocol of 10-15 watts to peak exercise with a target of exercise for 8-12 min. Exercise was terminated when the subject reached maximum capacity $\left(\mathrm{VO}_{2}\right.$ plateau; $85 \%$ of maximal heart rate $\left(\mathrm{HR}_{\max } ; 220\right.$-age $)$; respiratory quotient $\geq 1.1$; or self-reported exhaustion indexed via Borg Scale). A 3-min active recovery period completed the test. We used $\mathrm{VO}_{2}$ peak scores $(\mathrm{ml} / \mathrm{kg} / \mathrm{min})$ in all analyses.

\section{Social functioning}

We employed three complementary measures of SF including self-reports (Provision of Social Relations Scale, PSRS) [40], informant reports (Specific Levels of Functioning Scale, SLOF) [41], and clinician-administered interviews (Scale for the Assessment of Negative Symptoms, SANS) [33]. The PSRS is a 15-item self-report scale measuring social relationships with family and friends. Items are scored on a 5-point scale (from $1=$ "Very much like me" to $5=$ "Not at all like me") with higher scores indicating lower support and relationship quality. The PSRS was found to have alpha coefficients of $0.85,0.80$, and 0.78 among individuals with schizophrenia, bipolar disorder, and healthy controls, respectively [42], as well as solid test-retest reliability of $0.75-0.87$ [43]. The SLOF is a 43-item scale assessing multiple domains of daily functioning. Items are rated on a 5-point Likert scale with total scores ranging from 43 to 215 and higher scores indicating better overall functioning. The SLOF was found to have alpha reliability of 0.62 [41]. An informant version of the SLOF was administered to caregivers of all study participants, typically first-degree relatives who were in regular contact with study participants. For data analyses, we used the total score of the SLOF's Interpersonal Relations subscale. The SANS is a 25 -item clinician-administered scale developed to measure negative symptoms in schizophrenia, which have been linked to SF [44]. Items are rated on a 6-point scale (range 0-5), with higher scores reflecting increased severity. For the purposes of assessing SF, we used an Asociality subscale item from the SANS, which indexes the ability to feel intimacy and closeness with others (item \#20). The interrater reliability for this individual item of the SANS was found to be 0.90 [33]. 


\section{Demographic, clinical, and social network information}

Demographic and clinical information was collected including age, sex, primary language, reading ability, and baseline prescription of antipsychotic medication (as indexed by chlorpromazine equivalence). Additionally, we characterized the participants' social network, as indexed by item \#5 of the Heinrichs-Carpenter Quality of Life Scale (QLS) [45], a semi-structured interview. The item indexes the scope and degree of the participant's involvement in her/his social network - the extent to which other people concern themselves with the participant, care about her/his fortunes, or know about her/his activities.

\section{Study design and blinding}

We employed a proof-of-concept parallel assignment, single-blind RCT design with participants randomized to receive standard psychiatric treatment ("treatment as usual"; TAU) or attend a 12-week AE program in addition to TAU. Participants were randomized in the order they entered the study. After satisfying the inclusion/exclusion criteria, participants completed the diagnostic, clinical, aerobic fitness, and SF measures. Raters conducting clinical interviews and informant surveys, as well as the technicians conducting the aerobic fitness assessments, were blind to the participants' treatment status. Following the 12-week period, participants completed a follow-up assessment of clinical, aerobic fitness, and SF measures. Participants received \$270 for completing the research assessments. Additionally, those randomized to the AE arm received \$5 reimbursement for each AE session they attended (paid weekly) to defray the costs of round-trip public transportation.

\section{Interventions: description and fidelity assessments}

All participants received standard psychiatric care over the course of the study, which included regular meetings with a psychiatrist, psychologists, social workers, and/or psychiatric nurses as determined by their pre-study individual treatment plan. Treatment schedules were determined for each participant by their clinical team and no attempts were made to inform or influence treatment.

Participants randomized to the AE intervention underwent a 12-week, 3 sessions/week, 1-hour AE training program informed by the American College of Sports Medicine and federal guidelines, which recommend $150 \mathrm{~min}$ of moderate-intensity AE per week. Moderate-intensity AE involves activities that expend 3.0-5.9 times the energy expended at rest and are broadly defined as activities in which the participant is able to talk while engaging in the activity. All AE sessions were held in a small gym located in the medical center. The AE equipment included two treadmill machines, a stationary bike, an elliptical machine, and two active-play video game systems (Xbox 360 Kinect, Microsoft) with whole-body exercise software [29, 46]. The AE sessions were led by a certified exercise trainer (B.S. degree in Therapeutic Recreation). The sessions opened with a 10-min trainer-led warm-up period, followed by 45-min AE using the equipment, and ended with a 5-min cool-down period. The trainer was present during all AE sessions for guidance and support, along with a research assistant who assisted with equipment set-up and collection of AE-related behavioral data.

Training fidelity was indexed by 1) the number of AE sessions attended over the 12-week program (maximum 36) and 2) in-session AE intensity. The latter was set for participants individually based on their maximal heart rate $\left(\mathrm{HR}_{\max }\right)$, as determined during their baseline $\mathrm{VO}_{2}$ peak assessment. Target minimal AE intensity was set to $60 \%$ of $\mathrm{HR}_{\max }$ in Week $1,65 \%$ in Week 2, 70\% in Week 3, and 75\% in Weeks 4-12. In-session training intensity was monitored using Polar RS400 HR monitors (a wireless-enabled digital watch and chest strap) that participants wore during each session. The monitors were programmed to emit a soft beep when the participant's HR was lower than the individually targeted AE intensity level, which cued the trainer to encourage the participant to achieve their individual target goal.

Data Analyses: Data analyses were conducted using IBM SPSS ver. 25. All tests were two-tailed and the significance level was $\alpha=.05$. Determination of predictors of change in SF from baseline to 12-week follow-up was examined using three hierarchical step-wise regression analyses, using data from the PSRS, SLOF, and SANS as dependent variables. Change in aerobic fitness was entered in block 1 and demographic and clinical variables were entered in block 2 . Clinical and demographic variables included age, sex, baseline antipsychotic medication use, baseline anxiety and depression scores, and baseline social network. Shapiro-Wilk tests indicated that the primary dependent variables were normally distributed. The AE and TAU intervention groups were compared using multivariate analyses of variance with a repeated-measures design, with time and group designated as within-subject and between-subject factors, respectively. We focused our analyses on study completers, but also present results using an intention-to-treat approach with baseline observations carried forward.

\section{Results}

The sample's demographic and clinical characteristics are presented in Table 1. There were no significant group differences at baseline with regard to demographic, clinical, and physical health indices. There were also no significant group differences at baseline with regard to social functioning as 
Table 1 Baseline demographic and clinical characteristics

\begin{tabular}{|c|c|c|c|c|}
\hline & Aerobic exercise $(n=16)$ & Treatment as usual $(n=17)$ & $t / X^{2}$ & $p$ \\
\hline Age & $36.56(10.37)$ & $37.24(9.85)$ & .19 & .85 \\
\hline Sex (\% female $)$ & $37 \%$ & $35 \%$ & .02 & .89 \\
\hline Ethnicity (\% Hispanic) & $43 \%$ & $29 \%$ & .69 & .39 \\
\hline \multicolumn{5}{|l|}{ Race } \\
\hline Caucasian & $2(13 \%)$ & $6(35 \%)$ & 2.97 & .40 \\
\hline Black/African-American & $6(37 \%)$ & $6(35 \%)$ & & \\
\hline Asian & $2(13 \%)$ & $2(12 \%)$ & & \\
\hline More than one race & $6(37 \%)$ & $3(18 \%)$ & & \\
\hline \multicolumn{5}{|l|}{ Symptoms } \\
\hline Positive (SAPS global total) & $3.77(3.37)$ & $3.46(2.93)$ & .25 & .81 \\
\hline Negative (SANS global total) & $9.73(4.24)$ & $8.54(4.41)$ & .67 & .51 \\
\hline Depression (BDI total) & $7.81(7.69)$ & $7.23(8.57)$ & .20 & .84 \\
\hline Anxiety (BAI total) & $4.56(4.40)$ & $5.82(6.83)$ & .63 & .54 \\
\hline \multicolumn{5}{|l|}{ Medications } \\
\hline Antipsychotics (Chlorpromazine Equival.) & $258.85(232.51)$ & $439.73(362.78)$ & 1.69 & .10 \\
\hline Antidepressants (\% yes) & $44 \%$ & $35 \%$ & .25 & .62 \\
\hline SSRIs (\% yes) & $31 \%$ & $23 \%$ & .25 & .62 \\
\hline \multicolumn{5}{|l|}{ Social functioning } \\
\hline Self-report (PSRS) & $34.00(15.13)$ & $29.76(9.67)$ & .96 & .34 \\
\hline Informant (SLOF Interpersonal Total) & $4.75(2.52)$ & $3.65(2.76)$ & 1.20 & .24 \\
\hline Clinician (SANS Item \#20) & $2.06(1.39)$ & $1.76(1.44)$ & .61 & .55 \\
\hline Social Network (QLS Item \#5) & $3.56(1.46)$ & $4.00(1.94)$ & .73 & .47 \\
\hline Aerobic Fitness $\left(\mathrm{VO}_{2}\right.$ peak ml/kg/min) & $21.21(7.69)$ & $22.88(4.41)$ & .77 & .45 \\
\hline Body Mass Index & $31.60(6.57)$ & 30.75 (5.51) & .40 & .69 \\
\hline
\end{tabular}

$n=33$ (schizophrenia=26; schizoaffective disorder=7); SAPS, Scale for Assessment of Positive Symptoms; SANS, Scale for Assessment of Negative Symptoms; BDI, Beck Depression Inventory; BAI, Beck Anxiety Inventory; SSRI, Serotonin-Specific Reuptake Inhibitor; PSRS, Provision of Social Relations Scale; SLOF, Specific Level of Functioning-Informant version; SANS, Scale for the Assessment of Negative Symptoms; QLS, Quality of Life Scale; $\mathrm{VO}_{2}$ peak - maximum oxygen consumption

indexed by self-, informant-, and clinician-indexed ratings, as well as social networks. Additionally, there were no significant differences between groups in the number of clinical contacts with mental-health professionals (psychiatrists, psychologists, social workers, and/or psychiatric nurses) during the month prior to the 12-week follow-up assessments (AE: mean 5.00 meetings, SD 4.91 vs. TAU: mean 4.73, SD 6.09; $t=0.12, p=.91)$. Changes in SF over the course of 12 weeks were not associated with changes in prescription of antipsychotic, antidepressant, or SSRI medications. Likewise, no changes were reported in smoking status.

\section{AE training: safety, attendance, and in-session engagement}

Of the 16 participants assigned to the AE track, 13 successfully completed the training program $(81 \%)$. No serious adverse events were associated with the AE training. Participants attended 28.5 of the 36 scheduled AE sessions on average (79\%; range 44\%-97\%; 2.4 sessions per week on average). Two to three participants typically attended each
AE session. They exercised individually and were free to choose which AE equipment they wanted to use, as well as free to take breaks and hydrate as needed (breaks typically lasted 1-2 min).

During each AE session, participants engaged in active AE training on average for $42.7 \mathrm{~min} / \mathrm{session}$ (SD 3.51). Thirty-nine percent of this time was spent using the Xbox 360 Kinect, followed by the treadmill (32\%), stationary bike (13\%), elliptical machine (13\%), and trainer-led AE activities (3\%). On average, during $25 \%$ of the time during AE sessions, the participants' HR was between $60 \%$ and $69 \%$ of their $\mathrm{HR}_{\max }, 27 \%$ between $70 \%$ and $79 \%, 21 \%$ between $80 \%$ and $89 \%$, and $16 \%$ of the sessions at $\geq 90 \%$ of $\mathrm{HR}_{\max }$. During only $11 \%$ of the sessions was the participants' HR below the initial designated training goal of $60 \%$ of $\mathrm{HR}_{\max }$.

\section{Efficacy of aerobic exercise training to improve aerobic fitness}

Results on the impact of $\mathrm{AE}$ on aerobic fitness have been published previously [26]. Briefly, at follow-up, the AE 
Table 2 Predictors of change in self-, informant-, and clinician-reported social functioning in individuals with schizophrenia

\begin{tabular}{|c|c|c|c|c|c|c|c|c|}
\hline Dependent predictor variable & B & SE & $\operatorname{Beta}(\beta)$ & $p$ & $R^{2}$ & Adj. $R^{2}$ & $\Delta R^{2}$ & $\Delta$ Sig. $F$ \\
\hline \multicolumn{9}{|c|}{ Dependent variable: Change in self-reported social functioning (PSRS) } \\
\hline Step 1 & & & & & 0.22 & 0.18 & 0.22 & 0.02 \\
\hline Change in aerobic fitness & -1.18 & 0.46 & -0.46 & 0.017 & & & & \\
\hline Step 2 & & & & & 0.36 & 0.31 & 0.15 & 0.03 \\
\hline Change in aerobic fitness & -1.36 & 0.43 & -0.53 & 0.004 & & & & \\
\hline Age & -0.39 & 0.17 & -0.39 & 0.032 & & & & \\
\hline Step 3 & & & & & 0.47 & 0.39 & 0.10 & 0.05 \\
\hline Change in aerobic fitness & -1.84 & 0.46 & -0.72 & 0.001 & & & & \\
\hline Age & -0.41 & 0.16 & -0.40 & 0.018 & & & & \\
\hline Baseline social network (QLS) & -2.31 & 1.11 & -0.37 & 0.050 & & & & \\
\hline \multicolumn{9}{|c|}{ Dependent variable: Change in informant-reported social functioning (SLOF) } \\
\hline Step 1 & & & & & 0.07 & 0.03 & 0.07 & 0.19 \\
\hline Change in aerobic fitness & 0.27 & 0.20 & 0.26 & 0.194 & & & & \\
\hline Step 2 & & & & & 0.33 & 0.27 & 0.26 & 0.01 \\
\hline Change in aerobic fitness & 0.26 & 0.17 & 0.26 & 0.147 & & & & \\
\hline Baseline BAI & -0.36 & 0.12 & -0.51 & 0.007 & & & & \\
\hline \multicolumn{9}{|c|}{$\begin{array}{l}\text { Dependent variable: Change in clinician-rated social functioning (SANS item } \\
\text { 20) }\end{array}$} \\
\hline Step 1 & & & & & 0.07 & 0.03 & 0.07 & 0.19 \\
\hline Change in aerobic fitness & -0.08 & 0.06 & -0.27 & 0.186 & & & & \\
\hline Step 2 & & & & & 0.25 & 0.18 & 0.18 & 0.03 \\
\hline Change in aerobic fitness & -0.04 & 0.06 & -0.15 & 0.434 & & & & \\
\hline Baseline BAI & 1.03 & 0.44 & 0.44 & 0.029 & & & & \\
\hline
\end{tabular}

$n=33$; Aerobic fitness, $\mathrm{VO}_{2}$ peak $(\mathrm{ml} / \mathrm{kg} / \mathrm{min})$; PSRS Provision of Social Relations Scale, QLS Quality of Life Scale, SLOF Specific Level of Functioning, SANS Scale for the Assessment of Negative Symptoms; BAI Beck Anxiety Invent

participants improved their $\mathrm{VO}_{2} \max$ by $18.0 \%$ versus $-0.5 \%$ in the controls (group x time interaction, $F_{1,24}=12.88$; $p=.002)$.

\section{Predictors of improvement in social functioning}

Using a hierarchical step-wise regression analysis, we evaluated the impact of changes in AF and other predictor variables on SF. As shown in Table 2, the regression analysis indicated that for change in PSRS (i.e., self-reported SF), a model incorporating change in aerobic fitness, age, and baseline social network, significantly accounted for $47 \%$ of the explained variance in SF improvement $\left(F_{1,25}=6.395\right.$, $p=.003$ ), with change in aerobic fitness accounting for $22 \%$ of the explained variance $\left(F_{1,25}=6.619, p=.017\right.$; See Fig. 1). For change in SLOF (i.e., informant-reported SF), a model incorporating change in aerobic fitness and baseline anxiety significantly accounted for $33 \%$ of the explained variance in SF improvement $\left(F_{1,25}=5.617, p=.01\right)$. For change in SANS item 20 (i.e., clinician-rated SF), a model incorporating change in aerobic fitness and baseline anxiety significantly accounted for $25 \%$ of the explained variance in $\mathrm{SF}$ improvement $\left(F_{1,25}=3.824, p=.037\right)$.

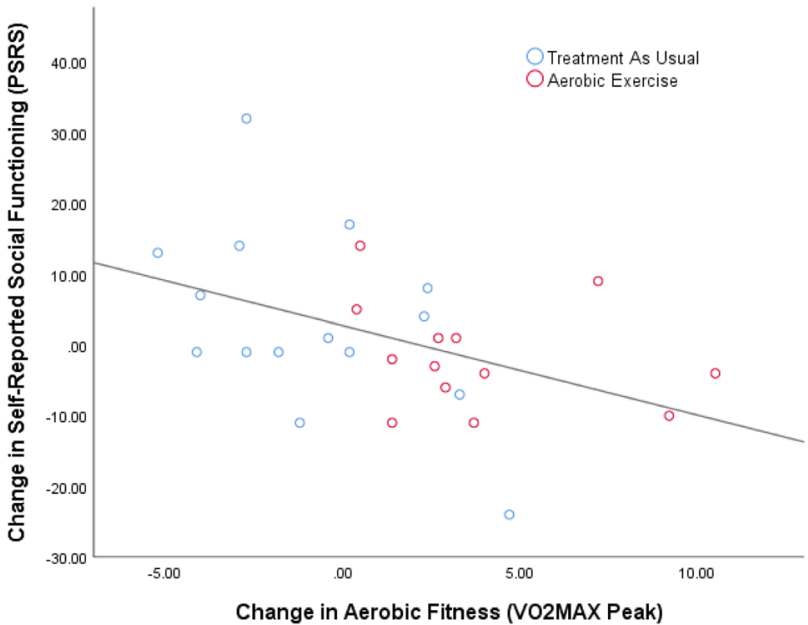

Fig. 1 Association of Changes in Aerobic Fitness and Changes in Social Functioning in Individuals with Schizophrenia. Note: $n=33$; PSRS-provision of Social Relations Scale; $\mathrm{VO}_{2}$ peak-maximum oxygen consumption 
Table 3 Comparison of changes in self-, informant-, and clinician-reported social functioning following aerobic exercise or treatment-as-usual

\begin{tabular}{|c|c|c|c|c|c|c|c|c|c|}
\hline \multirow[t]{2}{*}{ Treatment } & \multicolumn{4}{|c|}{ Intention to Treat $(\mathrm{n}=33)$} & \multicolumn{4}{|c|}{ Study Completers $(\mathrm{n}=26)$} & \multirow[b]{2}{*}{ Cohen's d ${ }^{b}$} \\
\hline & Mean $\Delta$ & SD & $\mathrm{F}^{\mathrm{a}}$ & $p$ & Mean $\Delta$ & SD & $\mathrm{F}^{\mathrm{a}}$ & $p$ & \\
\hline \multicolumn{10}{|l|}{ PSRS } \\
\hline TAU & 2.94 & 12.28 & 1.80 & 0.19 & 6.54 & 10.43 & 6.19 & $* 0.02$ & 0.89 \\
\hline $\mathrm{AE}$ & -1.31 & 6.83 & & & -1.62 & 7.60 & & & \\
\hline \multicolumn{10}{|l|}{ SANS } \\
\hline TAU & -0.76 & 5.33 & 0.54 & 0.47 & -0.46 & 2.07 & 0.32 & 0.58 & 0.19 \\
\hline $\mathrm{AE}$ & -1.00 & 5.74 & & & -0.85 & 1.99 & & & \\
\hline \multicolumn{10}{|l|}{ SLOF } \\
\hline TAU & -1.18 & 3.61 & 2.65 & 0.11 & -1.38 & 4.11 & 1.57 & 0.22 & 0.69 \\
\hline $\mathrm{AE}$ & 1.00 & 3.16 & & & 1.23 & 3.49 & & & \\
\hline
\end{tabular}

${ }^{*} p<.05 ; T A U$ treat-as-usual, $A E$ aerobic exercise, PSRS Provision of Social Relations Scale, SANS Scale for the Assessment of Negative Symptoms (Item 20); SLOF Specific Level of Functioning (informant version) - Interpersonal domain; Lower scores on the PSRS \& SANS and higher scores on the SLOF indicate better social functioning; ${ }^{a}$ Controlling for the impact of sex, age, and changes in antipsychotic medication; ${ }^{\mathrm{b}}$ For study completers

\section{Efficacy of AE training to improve social functioning}

Participants in the AE group who completed the study reported significant improvement in SF compared to the TAU group, as indexed by the PSRS $(23.0 \%$ vs. $-4.2 \%$; group $\times$ time interaction, $F_{1,24}=7.48, p=.012$; see Table 3 ). Results for the SANS and SLOF did not reach significance. Cohen's $d$ calculation indicated a large effect size for the AE intervention on the PSRS ( $d=0.89)$, a small effect size for the SANS asociality items $(d=0.19)$, and a medium effect size for the SLOF interpersonal subscale administered to caregivers $(d=0.69)$.

\section{Discussion}

To the best of our knowledge, the present report is the first comprehensive examination of the impact of AE on SF in individuals with schizophrenia. Our group had previously documented significant benefits of AE-related enhancement in aerobic fitness on neurocognition in this population [26]. Our results indicate that the benefits of enhancing aerobic fitness extend to SF, particularly as indexed by participants' self-reported experiences of social interactions. Notably, the models for informant- and clinician-rated SF provide additional support for the validity of our findings, though they were significant only after baseline level of anxiety was entered into the models.

As there are currently few effective treatments for social dysfunction in schizophrenia, our results, as well as the recent findings by Kern et al. [32], provide preliminary support for a novel intervention approach that target enhancement of aerobic fitness to improve poor SF in this population. This modality has advantages over available interventions in that it not only improves SF, but also enhances neurocognition [25, 26], cardiovascular functioning [29], as well as overall physical health. Additionally, our results suggest AE is a non-stigmatizing, safe, and nearly side-effect-free intervention, suggesting an intervention profile with a promise for broad dissemination. This view is consistent with the European Psychiatric Association's position paper and treatment guidelines advocating dissemination of physical activity as part of treatment for individuals with severe mental illness, [47] as well as previous reports for dissemination among fistepisode patients [48]. Additionally, within the context of the current COVID-19 pandemic, recent reports suggest AE may help protect against development of severe psychopathology [49].

Our results raise an important question about the mechanism underlying SF benefits. Specifically, whether, and to what extent, the SF benefits associated with AE were due to the increased availability of opportunities to engage in social interaction during AE sessions. While we cannot completely discount this explanation, it is unlikely given that typically the AE sessions involved only $2-3$ attendees per session; all participants exercised individually at different locations within the gym; and there were no attempts by staff to encourage social interactions between participants. Thus, participants typically had minimal interpersonal interactions during sessions. However, future studies should aim to formally assess this potential influence via use of an active control intervention. Our group [48] is currently examining this issue as part of a larger RCT employing AE and a stretching-and-toning, non-aerobic control intervention that offers identical opportunities for social interactions, as well as similar intervention duration, frequency, facilities, format (e.g., indoor space; small group), trainer contact, and financial compensation (i.e., reimbursement of exercise travel expenses), while not influencing aerobic fitness. 
Similarly, as the inclusion/exclusion criteria for the study excluded individuals with moderate or more severe symptoms of depression, it is unlikely that the results were driven by reduction in depressed mood. However, to control for this potential effect of baseline mood symptoms, we included both baseline anxiety and depression as covariates in our analysis. Conversely, previous reports indicate that emotional processing difficulties, particularly difficulties in emotion awareness and regulation, have been shown to be significant predictors of SF among individuals with schizophrenia and at clinical high risk for psychosis [22, 29, 50]. Thus, future studies should aim to examine whether the SF benefits of $\mathrm{AE}$ among individuals with schizophrenia-spectrum disorders are mediated by changes in emotion awareness and regulation.

Our results invite speculation about the putative neural circuitry that links AE-related changes in aerobic fitness and enhanced SF. Impairments in SF in schizophrenia have been attributed, in large part, to deficits in social cognition $[12,51]$. Specifically, deficits in the domains of emotion awareness and regulation, cognitive control, attribution, and theory of mind have been proposed to play a central role in determining SF in schizophrenia $[50,52,53]$. These domains have been linked to structural and functional abnormalities in the hippocampus, amygdala, ventral striatum, temporoparietal junction (TPJ), and posterior cingulate [54]. While data from imaging studies directly examining the link between $\mathrm{AE}$ and $\mathrm{SF}$ in schizophrenia are scarce, a number of recent reviews of studies employing structural MRIs have indicated volume increases in the hippocampus and cortical regions following exercise training $[27,55,56]$, although these findings are not universal. In particular, the hippocampus has been identified as a potential locus for illness severity and poor social functioning in schizophrenia [57], and research suggests that smaller hippocampal volume may result in increased stress sensitivity in first-episode schizophrenia [58]. Furthermore, pre-clinical and clinical studies have also indicated effects of AE on the hypothalamus-pituitary-adrenal axis, growth factors (e.g., Brain-Derived Neurotrophic Factor), immune-related mechanisms, as well as some effects on neurotransmitters and the endocannabinoid system [55].

Likewise, recent reports have linked a number of indices of cardiac autonomic regulation, which are positively responsive to $\mathrm{AE}$, to correlates of $\mathrm{SF}$ in individuals with schizophrenia. In particular, Hamilton and colleagues [59] demonstrated that respiratory sinus arrhythmia (RSA) and social cognition interact to predict functioning in this population. Specifically, RSA did not enhance functional outcomes when social cognition was already strong, but higher levels of RSA enabled effective role functioning when social-cognitive performance was impaired. Jointly, RSA and social cognition accounted for $40 \%$ of the variance in outcome success, compared with $21 \%$ when evaluating social cognition alone. More recently, our group [60] has documented links between lower autonomic arousal characterized by higher vagal input and metacognitive difficulties, which have been hypothesized to impact affective responses to social interactions [61]. These reports are consistent with reviews linking insular cortex functioning, which is heavily involved in autonomic arousal, and processing of social emotions and functioning [62]. Altogether, these findings are suggestive of a link between AE and enhanced neuroplasticity supporting improvement in SF. Future studies should confirm whether changes in these specific brain regions mediate the link between AE-related improvements in aerobic fitness and enhanced SF.

Our study has a number of strengths including a comprehensive, multi-method assessment of SF; state-of-the-art characterization of aerobic fitness; as well as granular, high temporal-resolution monitoring of in-session AE intervention fidelity. The latter is particularly important given that AE training characteristics (e.g., frequency, duration, and in-session intensity) may have significant impact on outcomes and thus are critical for design and dissemination of future intervention studies. AE training characteristics may also allow enhance replicability as well as allow comparison between studies with divergent findings. Our study has a number of limitations bear mentioning. First, the modest sample size suggests our results and conclusions should be viewed as preliminary. Future studies should aim to replicate our findings with larger samples. Second, the exclusion of individuals with schizophrenia with more than moderate symptoms of depression, as well as those regularly using substances, may potentially limit the generalizability of our findings. Third, our evaluations of SF all involved retrospective recollection of the participants' social activities by themselves (via self-reports and clinical interviews) and informants. Such evaluations are vulnerable to the influence of poor episodic memory, emotional states at the time of the evaluation, as well as cognitive biases and reframing [63]. This issue is particularly critical in studies of individuals with schizophrenia given the substantial episodic memory deficits documented in this population [64]. Consistent with these findings, our group has documented memory difficulties have a detrimental impact on accuracy of recollected experiences among individuals with schizophrenia [65]. To address this issue, future studies should aim to complement traditional retrospective assessments with ambulatory "in vivo, in situ" real-world evaluations of social interactions that would minimize the impact of reporting biases and offer higher ecological validity [66]. Finally, the lack of an active control group diminished our ability to examine the potential influence of opportunities for social contact on SF outcomes. Future studies should aim to examine the impact of AE on $\mathrm{SF}$ among such individuals. Our group recently started a 
large project that would be able to address some of these limitations [48].

In summary, our results indicate that $\mathrm{AE}$ is effective in improving SF in people with schizophrenia, particularly as indexed by study participants and their caregivers. Poor aerobic fitness represents a modifiable risk factor for social dysfunction in schizophrenia for which AE training offers a nonstigmatizing, safe, and nearly side-effect-free intervention.

\section{Compliance with ethical standards}

Conflict of interest On behalf of all authors, the corresponding author states that there is no conflict of interest.

\section{References}

1. Green CA, Fenn DS, Moussaoui D et al (2001) Quality of life in treated and never-treated schizophrenic patients. Acta Psychiatr Scand 103:131-142. https://doi.org/10.103 4/j.1600-0447.2001.00088.x

2. Addington J, Van Mastrigt S, Addington D (2004) Duration of untreated psychosis: impact on 2-year outcome. Psychol Med 34:277-284. https://doi.org/10.1017/S0033291703001156

3. Corcoran CM, Kimhy D, Parrilla-Escobar MA et al (2011) The relationship of social function to depressive and negative symptoms in individuals at clinical high risk for psychosis. Psychol Med 41:251-261. https://doi.org/10.1017/S0033291710000802

4. Robinson DG, Woerner MG, McMeniman M et al (2004) Symptomatic and functional recovery from a first episode of schizophrenia or schizoaffective disorder. Am J Psychiatry 161:473-479

5. Green MF, Bearden CE, Cannon TD et al (2012) Social cognition in schizophrenia, part 1: performance across phase of illness. Schizophr Bull 38:854-864. https://doi.org/10.1093/schbul/sbq17 1

6. Horan WP, Green MF, DeGroot M et al (2012) Social cognition in schizophrenia, part 2: 12-month stability and prediction of functional outcome in first-episode patients. Schizophr Bull 38:865-872

7. McCleery A, Lee J, Fiske AP et al (2016) Longitudinal stability of social cognition in schizophrenia: a 5-year follow-up of social perception and emotion processing. Schizophr Res 176:467-472

8. Jablensky A (2000) Prevalence and incidence of schizophrenia spectrum disorders: implications for prevention. Aust N Z J Psychiatry $34:$ S26-S34

9. Bellack AS, Green MF, Cook JA et al (2007) Assessment of community functioning in people with schizophrenia and other severe mental illnesses: a white paper based on an NIMH-sponsored workshop. Schizophr Bull 33:805-822

10. Burns T, Patrick D (2007) Social functioning as an outcome measure in schizophrenia studies. Acta Psychiatr Scand 116:403-418

11. Mueser KTE, Tarrier NE (1998) Handbook of social functioning in schizophrenia. Allyn \& Bacon, Needham Heights

12. Couture SM, Penn DL, Roberts DL (2006) The functional significance of social cognition in schizophrenia: a review. Schizophr Bull 32:S44-S63

13. Brissos S, Balanza-Martinez V, Dias VV et al (2011) Is personal and social functioning associated with subjective quality of life in schizophrenia patients living in the community? Eur Arch Psychiatry Clin Neurosci 261:509-517. https://doi.org/10.1007/s0040 6-011-0200-z
14. Revell ER, Neill JC, Harte M et al (2015) A systematic review and meta-analysis of cognitive remediation in early schizophrenia. Schizophr Res 168:213-222. https://doi.org/10.1016/j.schre s.2015.08.017

15. Devoe DJ, Farris MS, Townes P, Addington J (2019) Interventions and social functioning in youth at risk of psychosis: a systematic review and meta-analysis. Early Interv Psychiatry 13:169-180. https://doi.org/10.1111/eip.12689

16. Silva MJD, Cooper S, Li HL et al (2013) Effect of psychosocial interventions on social functioning in depression and schizophrenia: meta-analysis. Br J Psychiatry 202:253-260. https:// doi.org/10.1192/bjp.bp.112.118018

17. Laws KR, Darlington N, Kondel TK et al (2018) Cognitive Behavioural Therapy for schizophrenia-outcomes for functioning, distress and quality of life: a meta-analysis. BMC Psychology 6:32. https://doi.org/10.1186/s40359-018-0243-2

18. Louise S, Fitzpatrick M, Strauss C et al (2018) Mindfulnessand acceptance-based interventions for psychosis: our current understanding and a meta-analysis. Schizophr Res 192:57-63. https://doi.org/10.1016/j.schres.2017.05.023

19. Swartz MS, Perkins DO, Stroup TS et al (2007) Effects of antipsychotic medications on psychosocial functioning in patients with chronic schizophrenia: findings from the NIMH CATIE study. Am J Psychiatry 164:428-436

20. Sowa M, Meulenbroek R (2012) Effects of physical exercise on Autism Spectrum Disorders: a meta-analysis. Res Autism Spectrum Disord 6:46-57. https://doi.org/10.1016/j. rasd.2011.09.001

21. Bremer E, Crozier M, Lloyd M (2016) A systematic review of the behavioural outcomes following exercise interventions for children and youth with autism spectrum disorder. Autism 20:899-915

22. Kimhy D, Vakhrusheva J, Bartels MN et al (2014) Aerobic fitness and body mass index in individuals with schizophrenia: implications for neurocognition and daily functioning. Psychiatry Res 220:784-791

23. Vancampfort D, De Hert M, Myin-Germeys I et al (2017) Lower cardiorespiratory fitness is associated with more time spent sedentary in first episode psychosis: a pilot study. Psychiatry Res 253:13-17

24. Damme KS, Schiffman J, Ellman LM, Mittal VA (2020) Motor and Activity Psychosis-Risk (MAP-R) Scale: An exploration of scale structure with replication and validation. medRxiv

25. Firth J, Stubbs B, Rosenbaum S et al (2017) Aerobic exercise improves cognitive functioning in people with schizophrenia: a systematic review and meta-analysis. Schizophr Bull 43:546-556

26. Kimhy D, Vakhrusheva J, Bartels MN et al (2015) The impact of aerobic exercise on brain-derived neurotrophic factor and neurocognition in individuals with schizophrenia: a single-blind, randomized clinical trial. Schizophr Bull 41:859-868

27. Vakhrusheva J, Marino B, Stroup TS, Kimhy D (2016) Aerobic exercise in people with schizophrenia: neural and neurocognitive benefits. Curr Behav Neurosci Rep 3:165-175. https://doi. org/10.1007/s40473-016-0077-2

28. Nuechterlein KH, Ventura J, McEwen SC et al (2016) Enhancing cognitive training through aerobic exercise after a first schizophrenia episode: theoretical conception and pilot study. Schizophr Bull 42(Suppl 1):S44-S52. https://doi.org/10.1093/schbul/sbw007

29. Kimhy D, Lauriola V, Bartels MN et al (2016) Aerobic exercise for cognitive deficits in schizophrenia-the impact of frequency, duration, and fidelity with target training intensity. Schizophrenia Research

30. Fett A-KJ, Viechtbauer W, Penn DL et al (2011) The relationship between neurocognition and social cognition with functional outcomes in schizophrenia: a meta-analysis. Neurosci Biobehav Rev 35:573-588 
31. Dauwan M, Begemann MJH, Heringa SM, Sommer IE (2016) Exercise improves clinical symptoms, quality of life, global functioning, and depression in schizophrenia: a systematic review and meta-analysis. Schizophr Bull 42:588-599. https://doi. org $/ 10.1093 / \mathrm{schbul} / \mathrm{sbv} 164$

32. Kern RS, Reddy LF, Cohen AN et al (2020) Effects of aerobic exercise on cardiorespiratory fitness and social functioning in veterans 40 to 65 years old with schizophrenia. Psychiatry Res 291:113258. https://doi.org/10.1016/j.psychres.2020.113258

33. Andreasen NC (1989) Scale for the Assessment of Negative Symptoms (SANS). Br J Psychiatry 155:53-58

34. Andreasen NC (1984) Scale for the assessment of positive symptoms (SAPS). University of Iowa Iowa City

35. Beck AT, Steer RA, Brown G (1996) Beck depression inventoryII. Psychological Assessment

36. Steer RA, Beck AT (1997) Beck anxiety inventory

37. Ergometrics 800. SensorMedics, Inc., Yorba Linda, CA

38. Viasys Encore. Viasys Corporation, Loma Linda, CA

39. GE/Cardiosoft. Houston, TX

40. Turner RJ, Frankel BG, Levin DM (1983) Social support: Conceptualization, measurement, and implications for mental health. Accessed 3 Aug 2020

41. Schneider LC, Struening EL (1983) SLOF: a behavioral rating scale for assessing the mentally ill. Oxford University Press, Oxford, pp 9-21

42. Horan WP, Ventura J, Mintz J et al (2007) Stress and coping responses to a natural disaster in people with schizophrenia. Psychiatry Res 151:77-86

43. Huprich SK, Sanford K, Smith M (2002) Psychometric evaluation of the depressive personality disorder inventory. J Pers Disord $16: 255-269$

44. Ventura J, Subotnik KL, Gitlin MJ et al (2015) Negative symptoms and functioning during the first year after a recent onset of schizophrenia and eight years later. Schizophr Res 161:407-413. https://doi.org/10.1016/j.schres.2014.10.043

45. Heinrichs DW, Hanlon TE, Carpenter WT Jr (1984) The Quality of Life Scale: an instrument for rating the schizophrenic deficit syndrome. Schizophr Bull 10:388-398

46. Your Shape Fitness Evolved 2012. Ubisoft

47. Stubbs B, Vancampfort D, Hallgren M et al (2018) EPA guidance on physical activity as a treatment for severe mental illness: a meta-review of the evidence and Position Statement from the European Psychiatric Association (EPA), supported by the International Organization of Physical Therapists in Mental Health (IOPTMH). Eur Psychiatry 54:124-144. https://doi.org/10.1016/j. eurpsy.2018.07.004

48. Ospina LH, Wall M, Jarskog LF et al (2019) Improving Cognition via Exercise (ICE): Study Protocol for a Multi-Site, Parallel-Group, Single-Blind, Randomized Clinical Trial Examining the Efficacy of Aerobic Exercise to Improve Neurocognition, Daily Functioning, and Biomarkers of Cognitive Change in Individuals with Schizophrenia. J Psychiatr Brain Sci. https://doi. org/10.20900/jpbs.20190020

49. Mittal VA, Firth J, Kimhy D (2020) Combating the dangers of sedentary activity on child and adolescent mental health during the time of COVID-19. Am Acad Child Adolesc Psychiatry 59(11):1197-1198. https://doi.org/10.1016/j.jaac.2020.08.003

50. Kimhy D, Vakhrusheva J, Jobson-Ahmed L et al (2012) Emotion awareness and regulation in individuals with schizophrenia: implications for social functioning. Psychiatry Res 200:193-201. https://doi.org/10.1016/j.psychres.2012.05.029
51. Pinkham AE (2014) Social cognition in schizophrenia. J Clin Psychiatry

52. Dodell-Feder D, Tully LM, Lincoln SH, Hooker CI (2014) The neural basis of theory of mind and its relationship to social functioning and social anhedonia in individuals with schizophrenia. NeuroImage Clin 4:154-163

53. Fujiwara H, Yassin W, Murai T (2015) Neuroimaging studies of social cognition in schizophrenia. Psychiatry Clin Neurosci 69:259-267. https://doi.org/10.1111/pcn.12258

54. Stanley DA, Adolphs R (2013) Toward a neural basis for social behavior. Neuron 80:816-826. https://doi.org/10.1016/j.neuro n.2013.10.038

55. Maurus I, Hasan A, Röh A et al (2019) Neurobiological effects of aerobic exercise, with a focus on patients with schizophrenia. Eur Arch Psychiatry Clin Neurosci 269:499-515. https://doi. org/10.1007/s00406-019-01025-w

56. van der Stouwe ECD, van Busschbach JT, de Vries B et al (2018) Neural correlates of exercise training in individuals with schizophrenia and in healthy individuals: a systematic review. NeuroImage Clin 19:287-301. https://doi.org/10.1016/j.nicl.2018.04.018

57. Brambilla P, Perlini C, Rajagopalan P et al (2013) Illness severity and poor social functioning relate to hippocampal shrinkage in schizophrenia: a three-dimensional mapping study. Br J Psychiatry J Ment Sci 202:50

58. Smith GN, Lang DJ, Kopala LC et al (2003) Developmental abnormalities of the hippocampus in First-Episode schizophrenia. Biol Psychiatry 53:555-561. https://doi.org/10.1016/S0006 -3223(02)01977-7

59. Hamilton HK, Sun JC, Green MF et al (2014) Social cognition and functional outcome in schizophrenia: the moderating role of cardiac vagal tone. J Abnorm Psychol 123:764-770. https://doi. org/10.1037/a0037813

60. Ifrah C, Ospina LH, Shagalow S et al (2020) Cognitive insight and autonomic regulation during daily functioning in individuals with schizophrenia. Schizophr Res 218:300-301. https://doi. org/10.1016/j.schres.2020.03.013

61. Wells A, Matthews G (1996) Modelling cognition in emotional disorder: the S-REF model. Behav Res Ther 34:881-888. https:// doi.org/10.1016/s0005-7967(96)00050-2

62. Lockwood PL, Wittmann MK (2018) Ventral anterior cingulate cortex and social decision-making. Neurosci Biobehav Rev 92:187-191

63. Kimhy D, Delespaul P, Ahn H et al (2010) Concurrent measurement of "real-world" stress and arousal in individuals with psychosis: assessing the feasibility and validity of a novel methodology. Schizophr Bull 36:1131-1139. https://doi.org/10.1093/ schbul/sbp028

64. Berna F, Potheegadoo J, Aouadi I et al (2016) A meta-analysis of autobiographical memory studies in schizophrenia spectrum disorder. Schizophr Bull 42:56-66. https://doi.org/10.1093/schbu $1 /$ sbv099

65. Blum LH, Vakhrusheva J, Saperstein A et al (2015) Depressed mood in individuals with schizophrenia: a comparison of retrospective and real-time measures. Psychiatry Res 227:318-323. https://doi.org/10.1016/j.psychres.2015.03.008

66. Kimhy D, Vakhrusheva J, Vakhrusheva J (2019) Experience sampling in the study of psychosis. In: Experience sampling in mental health research. https://www.taylorfrancis.com/. Accessed $25 \mathrm{Aug}$ 2020 